\title{
EFEITO DA TEMPERATURA NA VISCOSIDADE DE ÓLEOS VEGETAIS REFINADOS
}

\section{EFFECT OF TEMPERATURE ON VISCOSITY OF REFINED VEGETABLE OILS}

\author{
César Augusto Canciam \\ Universidade Tecnológica Federal do Paraná - Campus Ponta Grossa \\ e-mail: ccanciam@hotmail.com
}

Recebido para publicação em: 12/01/2010

Aceite para publicação em: 01/04/2010

\section{RESUMO}

O conhecimento da viscosidade é um fator de fundamental importância para cálculos que envolvem a seleção de equipamentos e o dimensionamento de bombas e tubulações, assim como para a implementação de um efetivo controle de processos e garantia de qualidade do produto final. O objetivo deste trabalho foi estudar o comportamento reológico do azeite de oliva e dos óleos de arroz, canola, algodão, milho, girassol e soja nas temperaturas de 293,15 a $343,15 \mathrm{~K}$ $\left(20\right.$ a $\left.70^{\circ} \mathrm{C}\right)$, a partir da análise de regressão linear de dados experimentais encontrados na literatura. $\mathrm{O}$ efeito da temperatura na viscosidade foi expresso por relações do tipo Arrhenius, cujos coeficientes de correlação $\left(\mathrm{R}^{2}\right)$ foram superiores a 0,99 . As energias de ativação $(\mathrm{Ea})$ apresentaram, respectivamente, valores de $25,6213 \mathrm{~kJ} . \mathrm{mol}^{-1}, 26,2392 \mathrm{~kJ} . \mathrm{mol}^{-1}, 25,7215 \mathrm{~kJ} \mathrm{~mol}^{-1}, 27,4990 \mathrm{~kJ} . \mathrm{mol}^{-1}, 26,5298 \mathrm{~kJ}$. $\mathrm{mol}^{-1}, 27,1741 \mathrm{~kJ} \mathrm{~mol}^{-1} \mathrm{e} 27,7778 \mathrm{~kJ}$.mol para os óleos de soja, milho, girassol, arroz, algodão e canola e azeite de oliva, indicando que a maior sensibilidade da viscosidade em relação à mudança de temperatura está para o azeite de oliva.

Palavras-chave: Viscosidade. Temperatura. Óleos vegetais. Energia de ativação.

\begin{abstract}
The knowledge of viscosity is fundamental for calculations that involve the selection of equipments and the plotting of pumps and pipes, as well as for the implementation of effective processes control and warranty of quality of the final product. The objective of this work was to study the rheological behavior of olive, rice, canola, cotton, corn, sunflower and soybean oils in temperatures from 293,15 to $343,15 \mathrm{~K}\left(20\right.$ to $\left.70^{\circ} \mathrm{C}\right)$, starting from the analysis of lineal regression of experimental data found in the literature. The effect of the temperature in
\end{abstract}


the viscosity was analyzed by the Arrhenius relationships and the fitted models presented correlation coefficients $\left(\mathrm{R}^{2}\right)$ greater than 0,99 . The activation energies (Ea) calculated were, respectively, 25,6213kJ.mol ${ }^{-1}, 26,2392 \mathrm{~kJ} \cdot \mathrm{mol}^{-1}, 25,7215 \mathrm{~kJ}$. $\mathrm{mol}^{-1}, 27,4990 \mathrm{~kJ} \cdot \mathrm{mol}^{-1}, 26,5298 \mathrm{~kJ} . \mathrm{mol}^{-1}, 27,1741 \mathrm{~kJ} . \mathrm{mol}^{-1} \mathrm{e} 27,7778 \mathrm{~kJ} . \mathrm{mol}^{-1}$ for soybean oil, corn oil, sunflower oil, rice oil, cotton oil, canola oil and olive oil, indicating that the largest sensibility of the viscosity in relation to the temperature change is for olive oil.

Keywords: Viscosity. Temperature. Vegetable oils. Activation energy.

\section{INTRODUÇÃO}

Os óleos vegetais são constituídos predominantemente por triglicerídeos. Estão presentes, em menores quantidades, fosfolípideos, cerídeos, constituintes insaponificáveis, clorofilas e os produtos de alteração (FERRARI, 2001). Segundo Turatti (2000), os óleos vegetais são fontes de ácidos graxos na dieta humana. Os ácidos graxos são matérias-primas para a produção de energia através da $\beta$-oxidação em nível celular, necessária à realização de todos os processos de biossíntese pelo organismo humano.

Os lipídeos ainda desempenham importantes funções na fisiologia humana, dentre as quais se destacam as seguintes: participam da constituição de membranas celulares, organelas subcelulares e tecidos (principalmente, os tecidos adiposo e nervoso); agem como isolante térmico mantendo a temperatura corporal; promovem a proteção dos órgãos e da pele contra as radições cósmicas (tecido adiposo); promovem o amortecimento de choques físicos contra os órgãos (tecido adiposo) e são precursores na síntese de compostos como hormônios e lipoproteínas (TURATTI, 2000). Gioielli (1996) complementa que os lipídeos são precursores de ácidos biliares e moduladores celulares (como, por exemplo, leucotrienos, tromboxanos e protaglandinas) e fontes e veículos de vitaminas lipossolúveis (A, D, E e K).

Conceição e outros (2005) comentam que se tem verificado atualmente um aumento acentuado na demanda de mercado em relação a óleos vegetais das mais diversas fontes naturais, destacando-se as aplicações em derivados alimentícios que cobrem desde a formulação de produtos até a transformação e obtenção de ésteres a partir de triacilglicerídeos, ou, ainda, o emprego dos óleos vegetais diretamente na formulação de combustíveis.

A Tabela 1 relaciona a composição dos óleos de algodão, arroz, canola, girassol, milho e soja e do azeite de oliva em termos de ácidos graxos saturados, monoinsaturados e poliinsaturados. Os valores são expressos em gramas $(\mathrm{g})$ e determinados a partir de $100 \mathrm{~g}$ de cada tipo de óleo.

Tabela 1 - Composição dos óleos vegetais em termos da saturação de seus ácidos graxos.

\begin{tabular}{c|c|c|c}
\hline Espécie vegetal & $\begin{array}{c}\text { Ácidos graxos } \\
\text { saturados }(\mathrm{g})\end{array}$ & $\begin{array}{c}\text { Ácidos graxos } \\
\text { monoinsaturados }(\mathrm{g})\end{array}$ & $\begin{array}{c}\text { Ácidos graxos } \\
\text { poliinsaturados }(\mathrm{g})\end{array}$ \\
\hline $\begin{array}{c}\text { Algodão } \\
\text { (Gossypium ssp) }\end{array}$ & 25,50 & 19,55 & 53,05 \\
\hline Arroz (Oryza sativa) & 18,68 & 44,75 & 35,46 \\
\hline $\begin{array}{c}\text { Canola } \\
\left(\text { Brassica ssp) }{ }^{(1)}\right.\end{array}$ & 7,9 & 62,6 & 28,4 \\
\hline $\begin{array}{c}\text { Girassol } \\
(\text { Helianthus annuus) }\end{array}$ & 10,85 & 24,24 & 65,33 \\
\hline Milho (Zea mays) & 15,85 & 34,15 & 59,79 \\
\hline Soja (Glicine max) & 16,40 & 23,50 & 12,94 \\
\hline $\begin{array}{c}\text { Oliva } \\
\text { (Olea europaea) }\end{array}$ & 14,77 & 70,77 & 59,26 \\
\hline
\end{tabular}

Fonte: Tabela brasileira de composição de alimentos - USP (2009); (1) Tabela brasileira de composição de alimentos - TACO (2009).

De acordo com Marzzoco \& Torres (1990), as gorduras são ricas em ácidos graxos saturados e, por isso, à temperatura ambiente, são sólidas. Já os óleos vegetais são ricos em ácidos graxos insaturados e, dessa forma, à temperatura ambiente, são líquidos.

A viscosidade de um fluido mede a resistência interna oferecida ao movimento relativo das diferentes partes desse fluido (resistência ao fluxo). Conhecer e controlar essa propriedade é muito importante na formulação e preparação de emulsões, cremes, géis, soluções, entre outros produtos (SHAMES, 1999). 
No processamento de alimentos, a viscosidade é importante nas questões de escoamento de alimentos fluidos, agitação e mistura, perda de carga em tubulações e taxas de evaporação (CAMPOS et al., 1989).

Segundo Moura et al. (2000), o estudo da viscosidade é essencial para várias aplicações que incluem desde os projetos e avaliação de processos até o controle de qualidade, a correlação com a avaliação sensorial e a compreensão da estrutura dos materiais.

Pesquisas evidenciam a influência da concentração e da temperatura nos parâmetros reológicos, onde a relação do tipo Arrhenius geralmente é utilizada para descrever o efeito da temperatura na viscosidade de alimentos líquidos (GRATÃO, BERTO e SILVEIRA JÚNIOR, 2004).

Para Mafart \& Béliar (1994), a relação do tipo Arrhenius (Equação 1) é expressa em termos da temperatura absoluta $(\mathrm{T})$, da constante universal dos gases ideais $(\mathrm{R})$, da energia de ativação $(\mathrm{Ea})$, da viscosidade $(\eta)$ e de uma constante $\left(\eta_{0}\right)$, que, para Pereira, Queiroz e Figueirêdo (2003) é chamada de parâmetro de ajuste.

$$
\eta=\eta_{0} \cdot \exp (\mathrm{Ea} / \mathrm{R} \cdot \mathrm{T})
$$

Gratão, Berto e Silveira Júnior (2004) propõem escrever a relação do tipo Arrhenius na forma de:

$$
\ln \eta=\ln \eta_{0}+(\mathrm{Ea} / \mathrm{R}) \cdot(1 / \mathrm{T})
$$

A Equação 2 indica a dependência da viscosidade dos fluidos com a temperatura e a mesma pode ser utilizada com dados experimentais de viscosidade, na determinação dos parâmetros Ea e $\eta_{0}$. O conhecimento da energia de ativação (Ea) e do parâmetro de ajuste $\left(\eta_{0}\right)$ permite modelar matematicamente o comportamento reológico, bem como verificar os efeitos da temperatura sobre a viscosidade (GRATÃO, BERTO e SILVEIRA JÚNIOR, 2004).

A energia de ativação (Ea), segundo Pereira, Queiroz e Figueirêdo (2003), indica a sensibilidade da viscosidade devido à mudança de temperatura; maiores valores da energia de ativação significam que a viscosidade é relativamente mais sensível à mudança de temperatura.
Segundo Ribeiro et al. (2005), as equações mais utilizadas para o cálculo da viscosidade de líquidos baseiam-se na Teoria do Estado Ativado de Eyring (1941). Essa teoria considera que o líquido é formado por moléculas intercaladas por posições desocupadas (vacâncias), e essas moléculas, mesmo com o líquido em repouso, movem-se a fim de ocuparem as vacâncias adjacentes a elas. A viscosidade está relacionada com a força que tende a se opor a esse movimento, sendo uma medida de fricção interna do fluido. Assim, quanto maior a viscosidade, maior será a barreira potencial que uma molécula terá que vencer a fim de "saltar" para uma vacância adjacente. Essa barreira potencial é conhecida como energia de ativação.

O objetivo deste trabalho foi determinar os parâmetros Ea e $\eta_{0}$ para os óleos refinados de algodão, arroz, canola, girassol, milho e soja e para o azeite de oliva, com base em dados experimentais da viscosidade em função da temperatura encontrados na literatura, permitindo assim, a modelagem matemática do comportamento reológico para esses óleos vegetais.

\section{MATERIAIS E MÉTODOS}

A Tabela 2 indica os dados experimentais da viscosidade $(\eta)$ em função da temperatura absoluta (T) para os óleos refinados de algodão, arroz, canola e girassol, enquanto que a Tabela 3 indica os dados experimentais da viscosidade em função da temperatura absoluta para os óleos refinados de milho e soja e do azeite de oliva. Os dados fornecidos pelas Tabelas 2 e 3 foram adaptados de Brock et al. (2008).

Tabela 2 - Viscosidade dos óleos de algodão, arroz, canola e girassol em função da temperatura.

\begin{tabular}{c|c|c|c|c}
\hline T (K) & $\begin{array}{c}\text { Algodão (10 } \\
\mathrm{H}\end{array}$ & $\begin{array}{c}\text { Arroz }\left(10^{-3} \mathrm{~Pa} . \mathrm{s}\right) \\
\eta\end{array}$ & $\begin{array}{c}\text { Canola }\left(10^{-3} \mathrm{~Pa} . \mathrm{s}\right) \\
\eta\end{array}$ & $\begin{array}{c}\text { Girassol (10-3 Pa.s) } \\
\eta\end{array}$ \\
\hline 293,15 & 67,7 & 73,8 & 73,1 & 58,3 \\
\hline 303,15 & 47,3 & 50,5 & 50,5 & 41,3 \\
\hline 313,15 & 33,4 & 34,3 & 35,6 & 29,1 \\
\hline 323,15 & 24,6 & 24,5 & 25,2 & 21,3 \\
\hline 333,15 & 18 & 19,2 & 19,1 & 16,4 \\
\hline 343,15 & 14 & 14,2 & 14,5 & 12,6 \\
\hline
\end{tabular}

Fonte: Adaptado de Brock et al. (2008). 
Tabela 3 - Viscosidade dos óleos de milho e soja e do azeite de oliva em função da temperatura.

\begin{tabular}{c|c|c|c}
\hline \multirow{2}{*}{ (K) } & $\begin{array}{c}\text { Milho (10-3Pa.s) } \\
\mathrm{H}\end{array}$ & $\begin{array}{c}\text { Soja(10-3Pa.s }) \\
\eta\end{array}$ & $\begin{array}{c}\text { Oliva }\left(10^{-3} \text { Pa.s }\right) \\
\eta\end{array}$ \\
\hline 293,15 & 67,6 & 59 & 79,7 \\
\hline 303,15 & 47,4 & 41,2 & 55,4 \\
\hline 313,15 & 32,3 & 29,5 & 37,8 \\
\hline 323,15 & 24,8 & 22,3 & 26,2 \\
\hline 333,15 & 18,5 & 16,7 & 21,4 \\
\hline 343,15 & 14 & 12,6 & 14,9 \\
\hline
\end{tabular}

Fonte: Adaptado de Brock et al. (2008).

\section{RESULTADOS E DISCUSSÕES}

A Tabela 4 fornece os resultados obtidos pela análise de regressão linear para os óleos vegetais refinados.

A Tabela 5 fornece os valores para a energia de ativação $(\mathrm{Ea})$ e do parâmetro de ajuste $\left(\eta_{0}\right)$ com base nos valores indicados na Tabela 4 .

Tabela 4 - Resultados obtidos através da análise de regressão linear.

Considerando a constante universal dos gases ideais (R) igual a $8,314.10^{-3} \mathrm{~kJ} \cdot \mathrm{mol}^{-}$ $\mathrm{K}^{-1}$, foi possível determinar os parâmetros $\eta_{0}$ e Eados óleos vegetais refinados a partir dos dados indicados pelas Tabelas

\begin{tabular}{c|c|c|c|c|c|c|c}
\hline & Algodão & Arroz & Canola & Girassol & Milho & Soja & Oliva \\
\hline $\begin{array}{c}\text { Ea/R } \\
(\mathrm{K})\end{array}$ & 3190,9791 & 3307,5527 & 3268,4690 & 3093,7572 & 3156,0221 & 3081,7107 & 3341,0850 \\
\hline $\begin{array}{c}\ln \eta_{0} \\
(\mathrm{~Pa} . \mathrm{s})\end{array}$ & $-13,5812$ & $-13,9065$ & $-13,7714$ & $-13,4023$ & $-13,4706$ & $-13,3498$ & $-13,9305$ \\
\hline $\mathrm{R}^{2}$ & 0,9999 & 0,9991 & 0,9998 & 0,9997 & 0,9994 & 0,9999 & 0,9983 \\
\hline
\end{tabular}
2 e 3 e da análise de regressão linear para cada gráfico de $\ln \eta$ versus $1 / T$, onde o coeficiente angular da reta obtida de cada gráfico corresponde numericamente à razão $\mathrm{Ea} / \mathrm{R}$ e o coeficiente linear corresponde a $\ln \eta_{0}$.

Os valores da razão $\mathrm{Ea} / \mathrm{R}$ e de $\ln \eta_{0}$ foram obtidos, respectivamente, a partir das equações (3) e (4), as quais foram adaptadas de Triola (1999).

$$
\mathrm{Ea} / \mathrm{R}=\frac{\mathrm{n}[\Sigma(1 / \mathrm{T}) \cdot \ln \eta]-[\Sigma(1 / \mathrm{T})] \cdot(\Sigma \ln \eta)}{\mathrm{n} \cdot\left\{\Sigma\left[(1 / \mathrm{T})^{2}\right]\right\}-\{\Sigma(1 / \mathrm{T})\}^{2}}
$$

$\ln \eta_{0=} \underline{n} \cdot(\Sigma \ln \eta) . .\left\{\Sigma\left[(1 / T)^{2}\right]\right\}-[\Sigma(1 / T)] \cdot[\Sigma(1 / T) \cdot \ln \eta]$

$$
\text { n. }\{\Sigma[(1 / \mathrm{T})]\}-\{\Sigma(1 / \mathrm{T})\}^{2}
$$

A equação (5), também adaptada de Triola (1999), fornece o coeficiente de correlação $\left(\mathrm{R}^{2}\right)$ para a análise de regressão linear.

$$
\begin{aligned}
& \mathrm{R}^{2}= \\
& \mathrm{n}[\Sigma(1 / \mathrm{T}) \cdot \ln \eta]-[\Sigma(1 / \mathrm{T})] \cdot(\Sigma \ln \eta) \\
& \left\{\text { n. }\left\{\Sigma\left[(1 / \mathrm{T})^{2}\right]\right\}-\{\Sigma(1 / \mathrm{T})\}^{2}\right\}^{1 / 2} \cdot\left\{\mathrm{n} \cdot\left[\Sigma(\ln \eta)^{2}\right]-[\Sigma \ln \eta]^{2}\right\}^{1 / 2}
\end{aligned}
$$

Nas equações (3), (4) e (5), n representa o número de pares dados pelas Tabelas 2 e 3, ou seja, nesse caso $n$ é igual a 6 e $\Sigma$ denota a adição dos itens indicados.

Tabela 5 - Valores para a energia de ativação e para o parâmetro de ajuste.

\begin{tabular}{c|c|c}
\hline & $\mathrm{Ea}\left(\mathrm{kJ} . \mathrm{mol}^{-1}\right)$ & $\eta_{0}(\mathrm{~Pa} . \mathrm{s})$ \\
\hline Algodão & 26,5298 & $1,2640.10^{-6}$ \\
\hline Arroz & 27,4990 & $9,1303 \cdot 10^{-7}$ \\
\hline Canola & 27,1741 & $1,0451.10^{-6}$ \\
\hline Girassol & 25,7215 & $1,5117.10^{-6}$ \\
\hline Milho & 26,2392 & $1,4119.10^{-6}$ \\
\hline Soja & 25,6213 & $1,5932.10^{-6}$ \\
\hline Oliva & 27,7778 & $8,9138.10^{-7}$ \\
\hline
\end{tabular}

Com base na Tabela 5, pode-se observar que o azeite de oliva apresenta a maior energia de ativação, enquanto que o óleo de soja apresenta a menor energia de ativação.

Segundo Gratão, Berto e Silveira Júnior (2004), pesquisas evidenciam que os parâmetros reológicos sofrem a influência da concentração e da temperatura.

Com relação à concentração, com base na Tabela 1, o azeite de oliva e os óleos de arroz e de canola são óleos que apresentam maior concentração de ácidos graxos monoinsaturados. Como se pode observar na Tabela 5, esses óleos apresentam as maiores energias de ativação. Dessa forma, sugere-se que os parâmetros reológicos para os referidos óleos estão relacionados com a concentração de ácidos graxos monoinsaturados. 
Com relação à Tabela 1 , os óleos de milho, girassol e soja apresentam maior concentração de ácidos graxos poliinsaturados. De acordo com a Tabela 5, esses óleos apresentam as menores energias de ativação, o que sugere que os parâmetros reológicos para os mesmos estão relacionados com a concentração de ácidos graxos poliinsaturados.

Analisando as Tabelas 2 e 3, pode-se observar que em todos os intervalos de temperatura, a viscosidade do azeite de oliva foi maior do que a dos óleos de milho e de soja. De acordo com Ribeiro et al. (2005), com base na Teoria do Estado Ativado de Eyring (1941), quanto maior a viscosidade, maior será a barreira potencial que uma molécula terá que vencer a fim de "saltar" para uma vacância adjacente, ou seja, maior a energia de ativação. A análise de regressão linear confirma a maior energia de ativação, através da Tabela 5.

Com relação à temperatura, a própria equação do tipo Arrhenius indica a dependência da viscosidade com a temperatura, de maneira que o aumento da temperatura provoca uma diminuição da viscosidade. Essa redução de viscosidade com o aumento da temperatura é atribuída, segundo Grangeiro et al. (2007), ao aumento das distâncias intermoleculares provocadas pelo aquecimento. $\mathrm{O}$ aumento das distâncias reduz as forças atrativas entre as moléculas, diminuindo a viscosidade.

Associando a variação da temperatura com a energia de ativação, segundo Steffe (1996), citado por Toralles, Vendruscolo e Vendruscolo (2006), em geral uma alta energia de ativação implica que pequenas variações de temperatura são necessárias para modificar rapidamente a viscosidade. Assim, de acordo com a Tabela 5, os resultados sugerem que o azeite de oliva apresenta uma maior sensibilidade da viscosidade quanto ao aumento de temperatura, enquanto que o óleo de soja apresenta uma menor sensibilidade da viscosidade quanto ao aumento de temperatura, quando comparado aos outros óleos vegetais refinados.

Valores da energia de ativação $\left(E_{a}\right)$ e do parâmetro de ajuste $\left(\eta_{0}\right)$ para óleos vegetais refinados foram pesquisados na literatura para comparação, entretanto não foram encontrados.

\section{CONCLUSÕES}

$\mathrm{Na}$ faixa de temperatura de 20 a $70^{\circ} \mathrm{C}(293,15 \mathrm{a}$ $343,15 \mathrm{~K}$ ), foi possível determinar valores da energia de ativação (Ea) e do parâmetro de ajuste $\left(\eta_{0}\right)$, considerando a relação do tipo Arrhenius, para os óleos refinados de algodão, arroz, canola, girassol, milho e soja e do azeite de oliva. Para a determinação desses valores, utilizou-se a análise de regressão linear de dados experimentais, encontrados na literatura, de viscosidade e temperatura para as referidos óleos vegetais. A análise de regressão linear obteve um coeficiente de correlação $\left(\mathrm{R}^{2}\right)$ superior a 0,99 para todos os óleos vegetais.

Através de uma análise dos valores encontrados para a energia de ativação (Ea), os resultados sugerem que o azeite de oliva apresenta uma maior sensibilidade à mudança de temperatura em relação aos demais óleos vegetais, enquanto que o óleo de soja apresenta uma menor sensibilidade à mudança de temperatura em relação aos demais óleos vegetais.

Os resultados sugerem também que existe uma correlação entre a concentração dos ácidos graxos e os parâmetros reológicos dos óleos vegetais refinados. Óleos vegetais com maior concentração de ácidos graxos monoinsaturados apresentaram maiores valores para a energia de ativação, enquanto que óleos vegetais com maior concentração de ácidos graxos poliinsaturados apresentaram menores valores para a energia de ativação. Sugere-se que sejam realizados mais estudos confirmando, ou não, essa correlação.

O conhecimento da energia de ativação (Ea) e do parâmetro de ajuste $\left(\eta_{0}\right)$ para os óleos vegetais refinados permite modelar matematicamente o comportamento reológico desses óleos vegetais.

\section{REFERÊNCIAS}

BROCK, J. et al. Determinação experimental da viscosidade e condutividade térmica de óleos vegetais. Ciência e Tecnologia de Alimentos: Campinas, v.28, n.3, p.564-570.

CAMPOS, S.D.S. et al. Reologia e textura de alimentos. Campinas: ITAL, 1989. 84 p.

CONCEIÇÃO, M.M. et al. Rheological Behavior of Castor Oil Biodiesel. Energy \& Fuels: Washington, v.19, n.5, p. 2185-2188, 2005. 
FERRARI, R.A. Componentes minoritários de óleos vegetais. Óleos \& Grãos, São Paulo, n.58, p.20-28, 2001.

GIOIELLI, L.A. Óleos e gorduras vegetais: composição e tecnologia. Revista Brasileira de Farmacognosia: São Paulo, p.211-232, 1996.

GRANGEIRO, A.A. et al. Viscosidades de polpas concentradas de figo-da-Índia. Revista Brasileira de Agrociência: Pelotas, v.13, n.2, p.219-224, 2007.

GRATÃO, A.C.A.; BERTO, M.I.; SILVEIRA JÚNIOR, V. Reologia do açúcar líquido invertido: influência da temperatura na viscosidade. Ciência e Tecnologia de Alimentos: Campinas, v.24, n.4, p.652-656, 2004.

MAFART, P.; BÉLIARD, E. Ingeniería industrial alimentaria, volumen II: Técnicas de separación. Zaragoza: Editorial Acribia, 1994, $277 \mathrm{p}$.

MARZZOCO, A., TORRES, B.B. Bioquímica Básica. Rio de Janeiro: Editora Guanabara Koogan, 1990, 232 p.

MOURA, S.C.S.R. et al. Manual do Seminário Propriedades Termofísicas Aplicadas na Garantia da Qualidade dos Alimentos. Campinas: ITAL, 2000.

PEREIRA, E.A.; QUEIROZ, A.J.M.; FIGUEIRÊDO, R.M.F. Comportamento reológico de mel da abelha uruçu (Melípona scutellaris, $\boldsymbol{L}$.). Revista Ciências Exatas e Naturais: Guarapuava, v.5, n.2, p. 179-186, 2003.

RIBEIRO, L.D. et al. Cálculo de viscosidade de misturas não eletrolíticas. In: VI CONGRESSO BRASILEIRO DE ENGENHARIA QUÍMICA EM INICIAÇÃO CIENTÍFICA, 2005, Campinas. Disponível em: < http://www.feq.unicamp. br/ cobeqic/ttd07.pdf > . Acesso em: 12 out. 2009.

SHAMES, I.H. Mecânica dos Fluidos - volume 1. São Paulo: Editora Edgard Blücher, 1999. 192 p.

TABELA BRASILEIRA DE COMPOSIÇÃO DE ALIMENTOS - TACO. Disponível em: $<$ http://www.unicamp. $\mathrm{br} / \mathrm{nepa} / \mathrm{taco} /$ contar/taco_versao2.pdf $>$. Acesso em 12 out. 2009.

TABELA BRASILEIRA DE COMPOSIÇÃO DE ALIMENTOS - USP. Disponível em < http://www.fcf.usp.br/tabela/ lista.asp?base $=\mathrm{x}>$ Acesso em 12 out. 2009.

TORALLES, R.P., VENDRUSCOLO, J.L., VENDRUSCOLO, C.T. Reologia de Purê Homogeneizado de Pêssego: Efeito da Temperatura e Concentração. Brazilian Journal of Food Technology: Campinas, v.9, n.1, p.1-8, 2006.

TRIOLA, M.F. Introdução à Estatística. Rio de Janeiro: LTC Editora, 1999. 410 p.

TURATTI, J.M. Os lipídios na alimentação humana. Engenharia de Alimentos: São Paulo, n.33, p.23-26, 2000 